\title{
Neutron nuclear data measurements for criticality safety
}

\author{
Klaus Guber ${ }^{1, a}$, Carlos Paradela ${ }^{2}$, Jan Heyse ${ }^{2}$, Stefan Kopecky ${ }^{2}$, Peter Schillebeeckx ${ }^{2}$, and Peter Siegler ${ }^{2}$ \\ 1 Oak Ridge National Laboratory, PO Box 2008, Oak Ridge, TN 37831, USA \\ 2 Nuclear Physics Unit, EC-JRC, Retieseweg 111, 2440 Geel, Belgium
}

\begin{abstract}
To support the US Department of Energy Nuclear Criticality Safety Program, neutron-induced cross section experiments were performed at the Geel Electron Linear Accelerator of the Joint Research Center Site Geel, European Union. Neutron capture and transmission measurements were carried out using metallic natural cerium and vanadium samples. Together with existing data, the measured data will be used for a new evaluation and will be submitted with covariances to the ENDF/B nuclear data library.
\end{abstract}

\section{Introduction}

The US Department of Energy Nuclear Criticality Safety Program (NCSP) has a number of tasks requiring effective nuclear criticality safety calculations of fissionable material storage from former operations. Pertinent to these criticality calculations are improved neutron cross sections for nuclides involved in fissionable material conditioning and long-term storage. New data and evaluations including covariances are required for several stable fission products, as well as for materials found in mixtures with uranium. Evaluated data for cerium and vanadium found in the nuclear data libraries such as ENDF/B, JEFF, or JENDL do not perform well in criticality calculations [1]. In fact, a significant contribution to the neutron spectrum results from the epithermal energy region ( $\mathrm{keV}$ region), where the neutron cross section is sometimes not well known or non-existent. Identified deficiencies in nuclear data libraries have been outlined in the NCSP five-year plan. To respond to these needs, new neutron-induced cross section measurements have been initiated for cerium and vanadium to support the development of a new cross section evaluation in the resonance region.

\section{Experiments}

The Geel Electron Linear Accelerator facility (GELINA) [2] was used to perform neutron capture and transmission measurements. Currently, GELINA is the only time-offlight (TOF) facility left with excellent resolution over a broad neutron energy range.

The GELINA electron beam with a maximum energy of $150 \mathrm{MeV}$ is compressed to $1 \mathrm{~ns}$ pulse width using a bunching magnet. A pulsed electron beam is stopped in a rotating depleted uranium target, and the created Bremsstrahlung produces neutrons via photonuclear and photofission reactions. Two beryllium canned water containers mounted above and below the mercury-cooled uranium target aid as neutron moderators. Thus, the produced neutron spectrum ranges from thermal up to several $\mathrm{MeV}$; consequently, only a few experiments are

a e-mail: guberkh@ornl.gov needed to determine the cross section in the resolved and unresolved resonance regions. The combination of the small neutron production target, short neutron pulse, and long flight paths result in an excellent TOF resolution and thus neutron energy resolution. This facilitates resolving individual resonances in the neutron cross sections. For the reported experiments, GELINA operated at $800 \mathrm{~Hz}$ repetition rate and $1 \mathrm{~ns}$ pulse width.

The previous cerium experiments on which the actual evaluation relies were performed with cerium oxide samples. Using oxide as a target material has two significant drawbacks. First, the oxide is hygroscopic and contains water, so the exact chemical composition and number of atoms are uncertain. Secondly, for each cerium nucleus, there are two nuclei of oxygen that scatter mainly neutrons and can produce an unwanted prompt background in the capture experiments, which is very difficult to correct. For this reason, a natural metallic cerium sample was chosen and encapsulated in a thinwalled aluminium can to prevent reaction with air. Two disks with diameters of $5.985 \mathrm{~cm}$ and a thickness of $0.185 \mathrm{~cm}$ or $0.993 \mathrm{~cm}$, respectively, were produced for the experiments. The weight of the disks was $34.975 \mathrm{~g}$ and $187.945 \mathrm{~g}$. To compensate for the effect of the metallic can, data were taken with corresponding empty containers.

The vanadium experiments were performed with two metallic disks of natural composition and different thicknesses. The $0.22 \mathrm{~cm}$ thick disk had an average diameter of $8.0384 \mathrm{~cm}$ and a measured area of $50.7464 \mathrm{~cm}^{2}$ with a weight of $68.818 \mathrm{~g}$. The thin vanadium disk was $0.035 \mathrm{~cm}$ thick with a diameter of $8.0408 \mathrm{~cm}$, a measured area of $50.31369 \mathrm{~cm}^{2}$ and a weight of $10.503 \mathrm{~g}$.

\subsection{Transmission experiments}

Since the data for neutron capture cross sections are usually obtained with a rather thick sample, corrections must be applied by the data analysis programs. These corrections, including self-shielding and multiple scattering, can be quite sizeable and require reliable neutron widths as input to the data analysis programs. The lack of this information can result in erroneous capture

(C) The Authors, published by EDP Sciences. This is an Open Access article distributed under the terms of the Creative Commons Attribution License 4.0 (http://creativecommons.org/licenses/by/4.0/). 


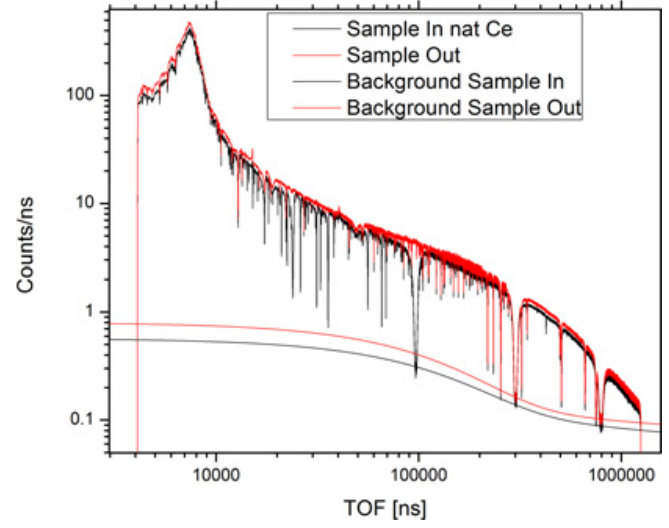

Figure 1. Example of the background correction for transmission data using black resonance filters.

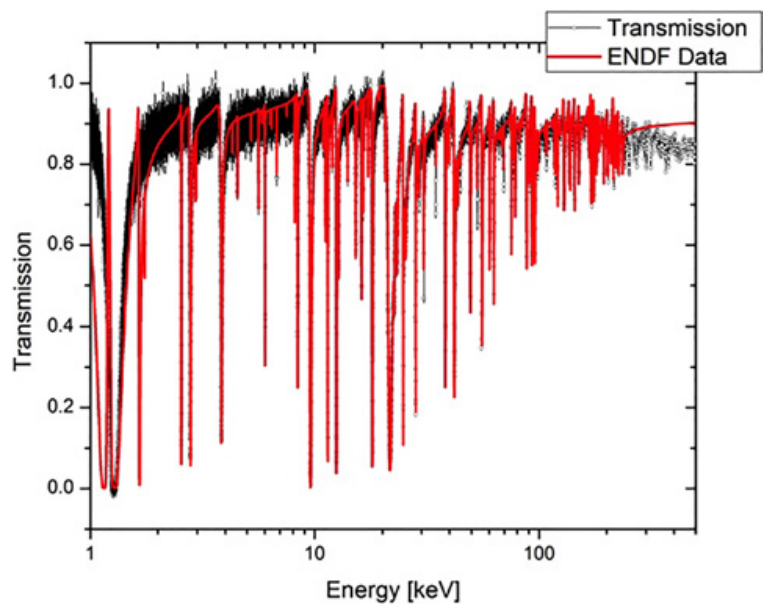

Figure 2. Transmission for thick natural cerium compared to calculated transmission using ENDF/B-VII.1 resonance parameters.

cross section data, as shown in Ref. [3]. For this reason, neutron transmission experiments were performed using all cerium and vanadium targets employing the flight path 4 experimental setup at a distance of $49.33 \mathrm{~m}$ from the neutron production target.

By inserting material (e.g., W, Co, Na and S) into the neutron beam, this so-called black resonance filter technique [4] is used to determine the time-dependent and -independent backgrounds for the transmission experiments. All neutrons are removed at certain neutron energies from the beam and are allowed to define the shape of the background. Typical uncorrected transmission spectra for the sample, open beam, and corresponding backgrounds are shown in Fig. 1.

A $0.635 \mathrm{~cm}$ thick ${ }^{6} \mathrm{Li}$-glass detector was used to detect the transmitted neutrons. A pre-sample collimator limited the neutron beam to a $4.5 \mathrm{~cm}$ diameter at the sample position in the $30 \mathrm{~m}$ flight station and ensured that neutrons did not affect the photomultiplier mounted on one side of the scintillation glass. To reduce the systematic uncertainties, the samples and their corresponding empty containers were cycled periodically through the neutron beam. As normalization, the total neutron count for each sample and cycle was recorded. The transmission spectrum for natural cerium and vanadium in the $\mathrm{keV}$ region are shown in Figs. 2 and 3, respectively.

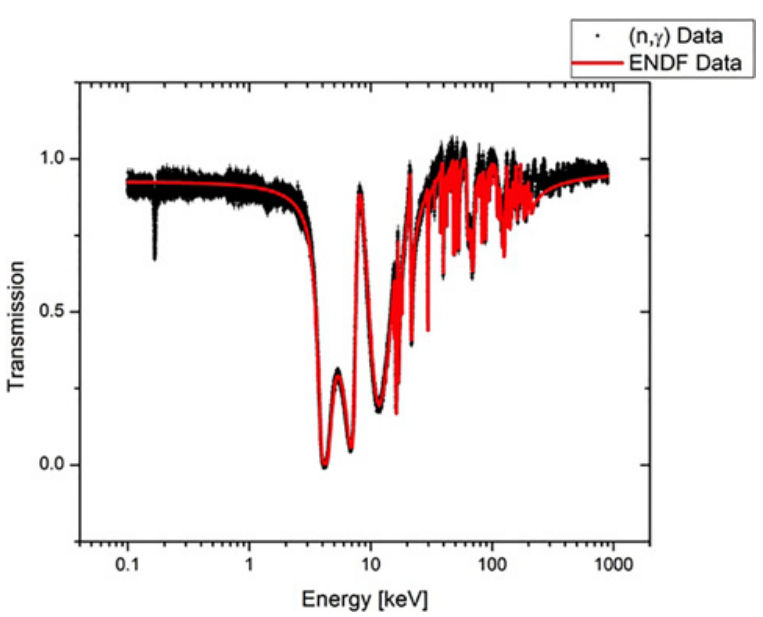

Figure 3. Transmission of the $2.2 \mathrm{~mm}$ thick sample vanadium compared to the calculated transmission using ENDF/B-VII.1 resonance parameters.

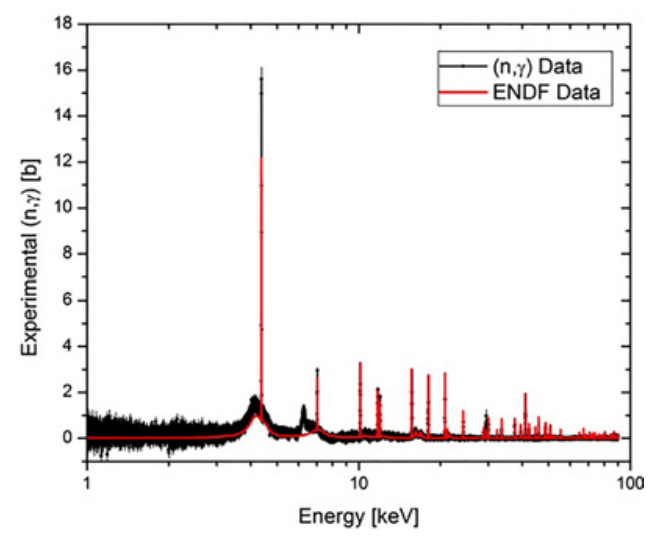

Figure 4. Detail view of the experimental neutron capture cross section for natural vanadium compared to the resonance parameters calculated from ENDF/B-VII.1.

\subsection{Neutron capture experiments}

The neutron capture data were obtained at the $60 \mathrm{~m}$ flight station on flight path 14 . Four $\mathrm{C}_{6} \mathrm{D}_{6}$-detectors mounted at an angle of $125^{\circ}$ to the neutron beam are registering the gamma ray after neutron capture in the sample. These so-called total energy detectors apply the pulse-height weighting method. By simulating the experimental set up, including the sample using MCNP, the weighting function was calculated. The cerium samples and corresponding empty container were mounted in the well-collimated beam on a low mass sample holder at a distance of $58.56 \mathrm{~m}$ from the neutron production target. For the vanadium capture experiments, disks of natural composition were mounted in the sample holder. Simultaneously, a ${ }^{10} \mathrm{~B}$ ionization chamber located in front of the sample was employed to record the neutron flux. For normalization of the capture data, an $80 \mathrm{~mm}$ diameter natural Fe sample was measured, and the well-known $1.15 \mathrm{keV}$ resonance in ${ }^{56} \mathrm{Fe}$ was used to obtain the normalization factor for the capture data. To simulate the sample scattered neutrons captured by the surroundings of the detector, a ${ }^{208} \mathrm{~Pb}$ sample was used. This effect is not negligible in the case of resonances with large scattering-to-capture ratio and for materials with small capture cross sections. Additional data were taken 


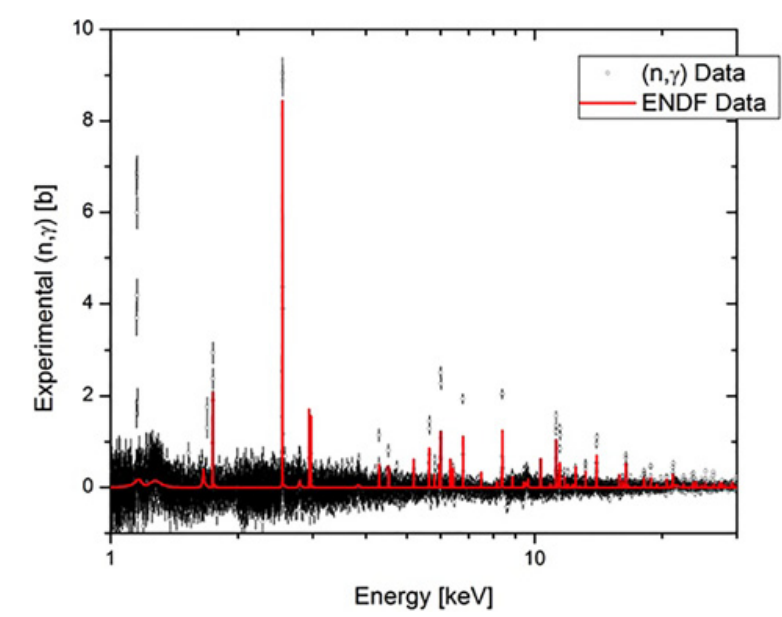

Figure 5. Neutron capture of natural cerium compared to the calculated capture cross section from ENDF/B-VII.1 resonance parameters.

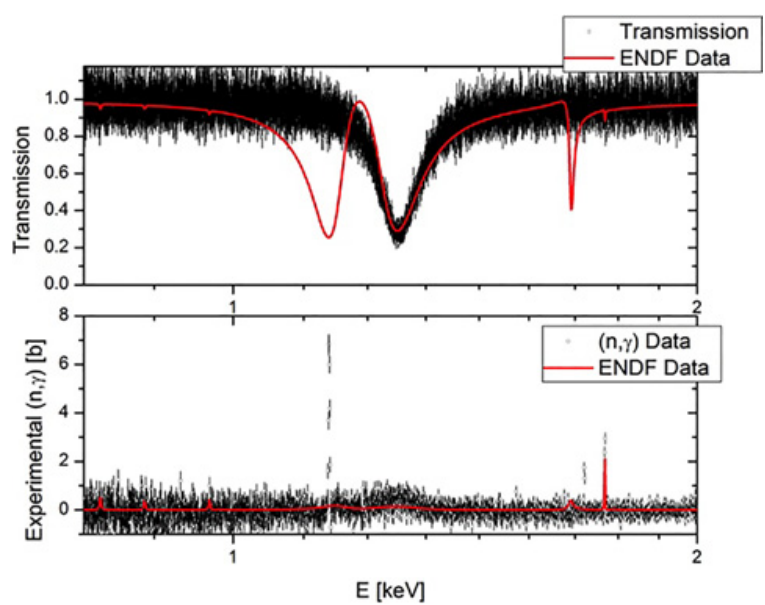

Figure 6. Neutron capture and corresponding transmission of natural cerium compared to calculated transmission and capture cross section using ENDF/B-VII.1 resonance parameters.

with various black resonance filters in the neutron beam to determine the beam-dependent backgrounds.

\section{Data reduction and analysis}

The data for transmission, neutron capture, and neutron flux are stored in list-mode; that is, for each event, TOF and pulse-height are recorded. Additionally, the corresponding scalers for each measurement's cycle are reported in an extra file. In the first step of the data sorting, the data for each run are adjusted for gain shift. In the second step, the individual runs are checked for stability using the information of different TOF windows and scalers. In the case of the neutron capture data, a weighting was applied using the pulse-height information. Subsequently, yield, cross section, or transmission is produced using the Analysis of Geel Spectra (AGS) software package [5]. AGS is capable of producing a covariance matrix for the recorded data; starting from counting statistics, it performs full uncertainty propagation.

The computer code SAMMY [6] developed at ORNL will be applied for the resonance analysis of the experimental data. This code uses the R-matrix formalism for treating the resonances and Bayes' theorem for the fitting process. In addition to the resonance parameters, a corresponding covariance matrix will be obtained from the analysis of the data sets. To test the data, a resonance parameter file for natural cerium and vanadium was constructed from the nuclear data library ENDF/B-VII.1 [7]. Input files for SAMMY describing the experimental condition and resonance parameters were created to compare the experimental data with the evaluated cross sections.

\section{Conclusions}

The shown cross section and transmission from ENDF/BVII.1 parameters are calculated using SAMMY, including all experimental effects such as multiple scattering, selfshielding, and resolution- and Doppler-broadening. The results are presented in Figs. 2-6 and demonstrate that the new capture and transmission data differ from the most current evaluated data file from the ENDF/B-VII.1 library. In fact, several resonances are missing or wrongly assigned in the evaluated nuclear data file even though they are reported in older experiments.

The reason for this discrepancy is twofold. First, the use of improper weighting functions resulted in mismatched detector response functions. In contrast to older experiments in which an arbitrary weighting function was used, this experiment used a Monte Carlo simulation with MCNP to determine the proper weighting function. Second, the not-well-understood neutron sensitivity of the older experimental setups led to capture cross sections that were erroneous. The inclusion of missing resonances in the new resonance parameter file will contribute to a higher cross section value.

An investigation of the significant difference shown in Fig. 6 for the cerium evaluation reveals that this discrepancy was introduced after the re-evaluation of the Working Party on International Nuclear Data Evaluation Cooperation (WPEC) subgroup 23 in 2005 [8]. This subgroup focused mainly on fission products, and it appears that in the case of the cerium, no literature or experimental data were consulted for comparison since all the correct information can be obtained. As a result, reevaluation without consulting the literature is not advised.

The findings in this series of experiments may have a significant impact on nuclear criticality calculations.

This manuscript has been authored by the Oak Ridge National Laboratory, managed by UT-Battelle LLC under Contract No. DE-AC05-00OR22725 with the U.S. Department of Energy. This work was supported by the Department of Energy (DOE) Nuclear Criticality Safety Program, funded and managed by the National Nuclear Security Administration for DOE. The United States Government retains and the publisher, by accepting the article for publication, acknowledges that the United States Government retains a non-exclusive, paid-up, irrevocable, worldwide license to publish or reproduce the published form of this manuscript, or allow others to do so, for United States Government purposes. The Department of Energy will provide public access to these results of federally sponsored research in accordance with the DOE Public Access Plan (http://energy.gov/downloads/doe-publicaccess-plan). 
This work was supported by the EUFRAT open access work package of the European Commission's Joint Research Centre EURATOM program under Horizon2020.

\section{References}

[1] http://ncsp.llnl.gov/planMain.html

[2] M. Flaska et al., Nuclear Instruments and Methods A 577, 626 (2004)

[3] P.E. Koehler et al., Physical Review C 54, 1463 (1996)
[4] D.B. Syme, Nuclear Instruments and Methods in Physics Research 198, 357 (1982)

[5] C. Bastian et al., Physor 2006, Vancouver, Canada (2006)

[6] N.M. Larson, ORNL Technical Report ORNL/TM9179/R8, October 2008

[7] M.B. Chadwick et al., Nucl. Data Sheets 112, 2887 (2011)

[8] https://www.oecd-nea.org/science/wpec/ volume23/volume23.pdf 